\title{
Kompatibilität aus der Perspektive des Designs
}

\author{
Marion Kießling' \\ Angenommen: 27. September 2021 / Online publiziert: 13. Oktober 2021 \\ (c) Der/die Autor(en) 2021
}

\section{Zusammenfassung}

Ergonomen wie Designer planen Mensch-Maschine-Systeme mit dem Ziel, den technischen Teil des Systems den menschlichen Eigengesetzlichkeiten anzupassen - Kompatibilität der Maschine mit dem Menschen herzustellen. Trotz des gemeinsamen Ziels gehen beide Disziplinen unterschiedlich mit dieser Aufgabe um und nutzen dafür unterschiedliche Begriffe. Hier werden die in der Gestaltung wichtigen Aspekte beschrieben: Was macht gute Kommunikation innerhalb des Mensch-Maschine-Systems aus? Was meinen Designer, wenn sie von der Funktion eines Produktes sprechen? Wie stellt sich die Paßgenauigkeit, das Maß der Kompatibilität, aus Sicht der Designer dar? Wie werden Erkenntnisse aus der Gestaltpsychologie und der Wahrnehmungspsychologie umgesetzt?

Gestalter gehen insbesondere auf die emotionalen, gesellschaftlichen und ästhetischen Bedürfnisse der Nutzer ein. Sie beschäftigen sich mit der Vorstellung, dem Konzept des Nutzers von dem Objekt, mit dem er interagiert und versuchen ihn dort abzuholen.

Je genauer diese Passung mit Einzelprodukten und ganzen Systemen gelingt, umso besser ist die Akzeptanz und der Anreiz, die Mensch-Maschine-Systeme auszureizen, ja zu genießen.

Ästhetik betrifft die Wahrnehmung und die Entscheidungsprozesse. Sie ist keine oberflächliche Dreingabe, die Designer einem technischen Produkt aufsetzen, sondern berühren die fundamentalen Bedürfnisse der Menschen. Diese Produkte funktionieren schön, sie weisen eine um die ästhetischen Aspekte erweiterte Kompatibilität auf.

Praktische Relevanz: Technische Kompatibilität kommt erst zum Tragen, wenn das Gerät wirklich benutzt wird. Genutzt wird es, wenn der Nutzer über die praktische Funktion hinaus einen emotionalen Mehrwert (Joy of Use) vermutet.

Schlüsselwörter Kompatibilität Interface $\cdot$ Kommunikation $\cdot$ Joy of Use $\cdot$ Produktfunktionen $\cdot$ Reduktion der Komplexität $\cdot$ Gestaltgesetze $\cdot$ Mentales Modell $\cdot$ Multimodalität $\cdot$ Kontingenz $\cdot$ Konsistenz $\cdot$ Ästhetik

\section{Compatibility from the perspective of design}

\section{Abstract}

Human Factors Engineering specialists as well as designers develop Human-Machine-Systems with the aim of fitting the technical component of the system to man's inherent properties, that is, to create compatibility between man and machine. Despite a common goal, both disciplines deal with this task differently, as well as using differing terms. This paper provides considerations which are relevant to Design:

What constitutes good communication within a Human-Machine-System? What does the designers' term function signify? How is fitting accuracy to be characterized, a measure for compatibility? How are the findings from Gestalt Psychology and Cognitive Psychology implemented in a product?

Designers respond particularly to the emotional, social and aesthetic needs of users. They strive to meet the user's expectations by referring to the user's mental model of the object interacted with. The better the match is achieved with single products and entire systems, the higher will be their acceptance and the incentive to fully use them, even to enjoy them.

Prof. Marion Kießling marion.kiessling@hm.edu
1 Fakultät für Design, Hochschule München, Lothstraße 17, 80335 München, Deutschland 
Aesthetics involve our perception and our decisions. It is not a superficial bonus which designers lend to technical products. It rather touches on our fundamental needs. These products work beautifully, as they exhibit extended compatibility, extended by aesthetic aspects.

Practical relevance: Compatibility only comes into effect, when the compatible product actually is being used. It will be used, if a user assumes to enjoy an emotional added value (Joy of Use) on top of the product's purpose.

Keywords Compatibility interface $\cdot$ Communication - Joy of use $\cdot$ Product functions · Reducing complexity $\cdot$ Gestalt principles $\cdot$ Mental model $\cdot$ Multimodal $\cdot$ Contingency $\cdot$ Consistency $\cdot$ Aesthetics

\section{Einleitung}

Im Mensch-Maschine-System treten die beiden Korrespondenten ${ }^{1}$ Mensch und Maschine in eine funktionale Beziehung,

bilden in ihrer Verschiedenheit eine Einheit. Diese Beziehung muß konzipiert und gestaltet werden. Ziel dieser Gestaltungsaufgabe ist, den Austausch zwischen den Korrespondenten zu ermöglichen und zu optimieren - Kompatibilität zu entwickeln.

Im Folgenden wird dargestellt, welche Aspekte und Zielsetzungen Designer bei dieser Aufgabe beachten. Das soll an Beispielen aus der Gestaltungspraxis und Lehre erläutert werden.

\section{Ergonomie und Design}

Ähnlich der Ergonomie ist Design eine Disziplin, die sich auf Erkenntnisse aus vielen Wissensgebieten beruft. Themen werden sowohl von Ergonomen als auch von Designern behandelt, wenn auch aus unterschiedlichen Perspektiven betrachtet.

Die Kommunikation zwischen Mensch und Maschine setzt voraus, daß sich die beiden verstehen. Da sie jedoch grundsätzlich unterschiedliche Sprachen (Codes) verwenden, bedarf es einer Übersetzung. Auf dem Weg vom Bearbeitungswunsch des Menschen bis zur Darstellung des Ergebnisses durch die Maschine erfährt die jeweils spezifisch codierte Information eine Reihe von Umcodierungen (vgl. Abb. 1).

So ist eines der von Ergonomen beschriebenen Ziele, einen möglichst geringen Umcodieraufwand bei der Informationsaufnahme abzuverlangen, analog zum Bestreben der Designer nach guter Kommunikation der Geräte mit dem Nutzer. Mit guter Kommunikation meinen Designer eine Kommunikation, die sowohl effektiv und effizient ist, als auch Freude bereitet (Joy of Use). Dieser, die Emotionen ansprechende Aspekt, geht über die Zielsetzung der Ergonomen hinaus.

\footnotetext{
${ }^{1}$ Hier wird anstelle des sonst üblichen Begriffs Subsystem der Begriff Korrespondent verwendet, da die kommunikativen Aspekte des Mensch-Maschine-Systems betrachtet werden.
}

Claude Toussaint, Ingenieur und Designer, beschrieb diesen Unterschied zwischen den Zielen der Ergonomen und denen der Designer mit einer Analogie: Der Designer ist der Koch, der aus dem Kalorienlieferanten einen Genuß machen kann. Der Ergonom ist der Ernährungswissenschaftler, der weiß, was die Grundbedürfnisse des Menschen sind.

Für den Designer Kenya Hara (2011) ist Design weniger um Wohlfühlen als um Anregung bemüht. Er definiert Design sogar als die energische Anerkennung unserer eigenen Lebenswelt, indem wir Dinge bauen und indem wir kommunizieren. Er sieht Design als Provokation der Sinne, als Anlaß, Dinge neu zu sehen. Auch wenn uns nicht jedes der Produkte, die wir nutzen, so positiv provoziert, bereichern sie uns doch mit der Gelegenheit, sie zu analysieren und eine wie auch immer geartete Beziehung zu ihnen zu entwickeln.

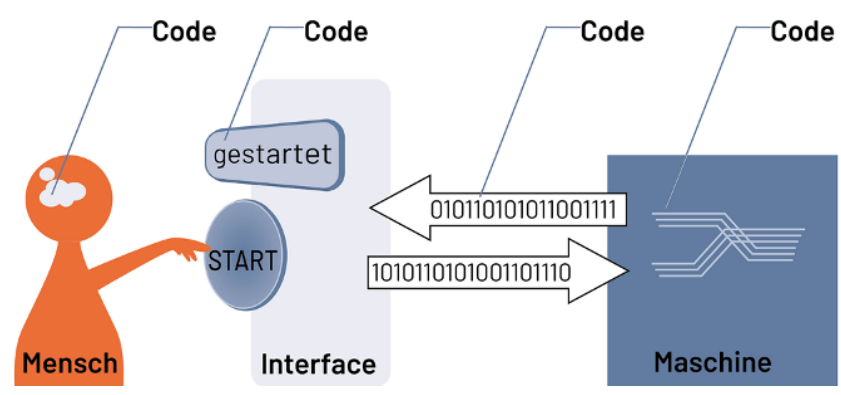

Abb. 1 Umcodierung bezeichnet die Übersetzung zwischen den Ausdrucksformen verschiedener Agenten. Am Beispiel des Mensch-Maschine-Interfaces wird mehrfach umcodiert: Zunächst wird die interne Sprache des Menschen in eine Handlung übersetzt, eine Eingabe in das Interface. Das Interface setzt diese Eingabe in Maschinencode um, der in der ausführenden Maschine zu interner Kommunikation und entsprechenden Aktionen führt. Als Feedback übermittelt die Maschine eine Nachricht an das Interface, das diesen Code als für den Nutzer verständliche Information darstellt

Fig. 1 Code conversion refers to the translation between the expressions of various agents. Using the example of human-machine interfaces, multiple code conversions take place: At first, a person's internal idiom is converted into an action, turning an intention into the activation of a control on the interface. The interface then transforms this signal into machine code, which in turn has the executing machine to relay internal communication, triggering appropriate action. The machine transmits a message to the interface, which then displays this code as comprehensible information for users 
Der Wissenschaftsjournalist Patrick W. Jordan (2000) setzt sich mit Produkten aus der Nutzerperspektive auseinander. Er differenziert vier Arten der Freuden, die ein Produkt zum Lieblingsobjekt machen:

- Das sensuale Vergnügen (Physio-pleasure), das über die Sinne wahrgenommen wird, wie z. B. der Kaschmirpullover oder guter Wein;

- das soziale Vergnügen (Socio-pleasure), das Bekannte vereint und Identifikationsmerkmal der Gruppe ist, wie dies beispielsweise ein Kaffeeautomat oder eine Sportart auslöst;

- das psychische Vergnügen (Psycho-pleasure) durch die effiziente und intuitive Art der Unterstützung durch das Produkt, die beispielsweise eine reibungslose Buchung ermöglicht oder ein leichtgängiges Fahrrad;

- die Freude am Ästhetischen (Ideo-pleasure), die Wertigkeit, die dem ideellen Wert des Produktes zugewiesen wird, wie z. B. ein geerbtes Schmuckstück oder ein Modell des F1-Siegerfahrzeugs, das man live sah.

Grundlage solcher Produkte, die im Sinn der Designer gut kommunizieren, ist die Kompatibilität der Bedürfnisse, Erwartungen und Fähigkeiten der Nutzer mit den Produkten. Neben dieser Paßgenauigkeit wird immer auch angestrebt, die Komplexität eines Produktes bzw. eines Systems zu reduzieren.

\section{Begriffe in der Ergonomie - Begriffe im Design}

Vorab, um Mißverständnissen vorzubeugen, sollen einige Design-Begriffe erläutert werden: Anstatt des Menschen wird im Design der (meist menschliche) Nutzer betrachtet. Die Maschine im Mensch-Maschine-System ist ein (zu gestaltendes) Produkt oder ein Service.

\subsection{Funktionen eines Produktes}

Auch für die Funktionen eines Produktes gibt es eigene Bezeichnungen, die über die praktischen Funktionen, den Zweck eines Produktes, hinausgehen. Designer kümmern sich entsprechend nicht nur darum, daß beispielsweise der Küchenmixer auch mixt, also einen Zweck erfüllt (gemäß dem überholten Slogan form follows function), sondern bemühen sich u. a. auch um die formalästhetischen Funktionen und um Anzeichen- sowie um Symbolfunktionen des Mixers (vgl. Abb. 2).

$\mathrm{Zu}$ den formalästhetischen Funktionen des Mixers zählen formale Kriterien wie Proportionen, Linienqualität etc., die die Form an sich zum Gegenstand haben.

Formalästhetische Eigenschaften können den Nutzer durch seine ästhetischen Aspekte erfreuen, das Produkt attraktiv erscheinen lassen.

Anzeichenfunktionen zeigen an, um welche Produktart es sich handelt und was man damit tun kann. Anzeichenfunktionen geben auch Hinweise auf gefährliche Bereiche, ähnlich einem Warnhinweis „Nicht anfassen!“”. Zugleich markieren bestimmte Anzeichen die ungefährlichen Bereiche und laden sogar dazu ein, das Produkt hier, am ausgewiesenen Handteil, anzufassen (vgl. Abb. 3).

Auch die weitere Interaktion mit dem Produkt wird durch derartige Hinweise unterstützt, von der Aktivierung bis zur sicheren Handhabung. Anzeichenfunktionen bewirken, daß der Nutzer erkennt, was das Produkt leistet, wie es funktioniert bzw. wie es genutzt wird und in welchem Zustand es sich gerade befindet. Dies entspricht der in der Ergonomie geforderten Reiz-Reiz-Kompatibilität, nach der der Benutzer eine bestimmte Beziehung zwischen Anzeige und Wirklichkeit erwartet (vgl. Abb. 4).

Als eine der zeichenhaften Funktionen schreiben die Symbolfunktionen kontextuale Bedeutungen zu: Welche soziale Funktion erfüllt das Produkt, d.h. wie stellt es den gesellschaftlichen Status, den Wohlstand, die Technikaffinität des Nutzers dar? Auch der zeitliche Kontext spielt eine Rolle, nach dem die Aktualität des Produktes bewertet wird: Ist das Produkt traditionell oder avantgardistisch? Der
Abb. 2 Klassifizierung der Produktfunktionen gemäß dem Offenbacher Ansatz (vgl. Steffen 2000)

Fig. 2 Classification of the product functions according to the Offenbacher Ansatz, (see Steffen 2000)

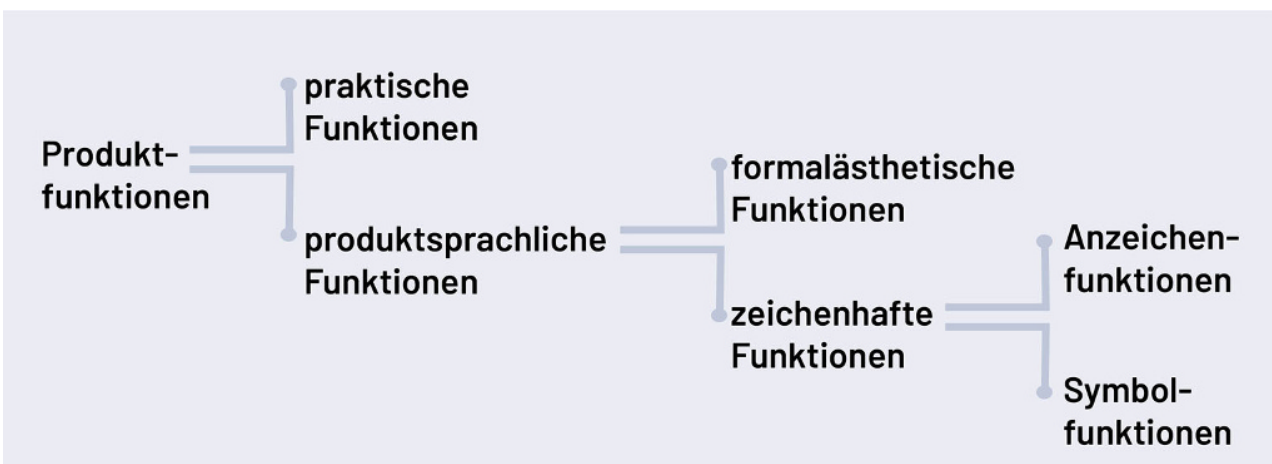




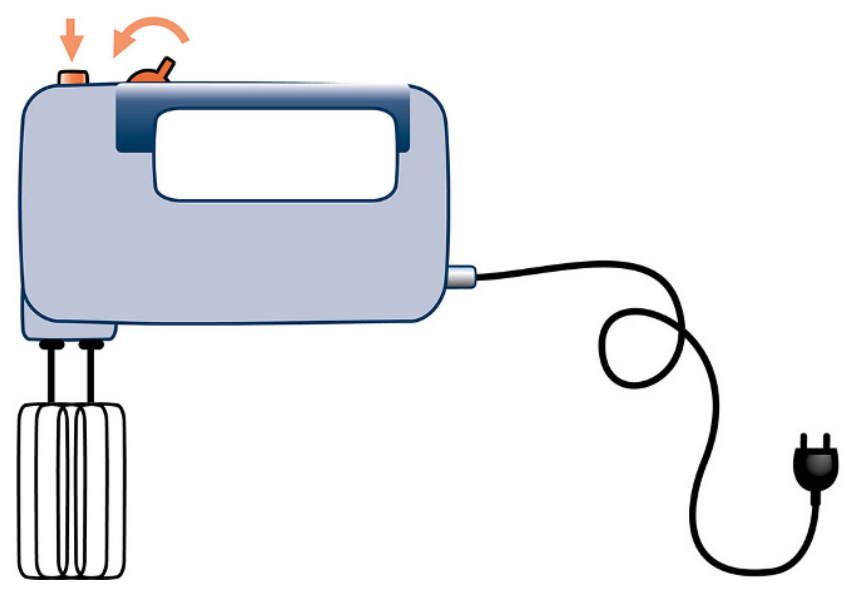

Abb. 3 Auszeichnung von ungefährlichen bzw. gefährlichen Bereichen auf einem Küchenquirl

Fig. 3 Indication of a kitchen mixer's safe respectively hazardous contact areas

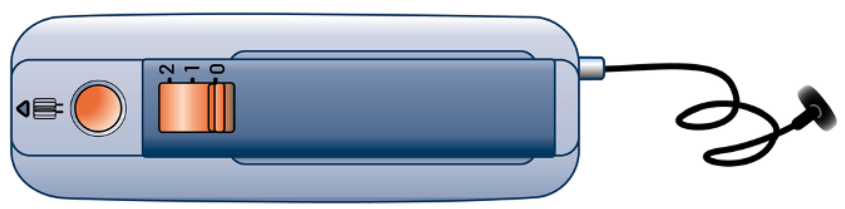

Abb. 4 Form und Lage der Bedienelemente geben Hinweise zum Status und zur Handhabung des Geräts

Fig. 4 Shape and location of the controls indicate status and provide advice for handling the device

Markenkontext, das Branding des Produktes, steht für die Selbstdarstellung des Nutzers, charakterisiert ihn gemäß der Markenidentität. Wie wichtig uns diese Symbolfunktionen tatsächlich sind, kann man z. B. an Diskussionen um Kleidungsstile erkennen.

Die Gesamtheit der Produktfunktionen gibt das Ziel des Gestalters vor. Die Produktfunktionen sollten einen stimmigen Gesamteindruck ergeben, d.h. konsistente Sinneseindrücke, die sich z.B. aus dem visuellen Eindruck und dem Abgleich mit dem Eindruck von Gewicht, Geräusch, Bewegung, dem olfaktorischen und dem haptischen Eindruck ergeben. Dieses Ziel ist die Anmutung, der Eindruck, den das gestaltete Produkt vermitteln sollte und gleichzeitig der Ausdruck, der dem Produkt mitgegeben wird. Im Idealfall ist das Produkt so gestaltet, daß es nicht nur intuitiv benutzbar ist, sondern darüber hinaus dem Nutzer ästhetischen Genuß bereitet und ihn mit Besitzerstolz erfüllt - der höchsten Stufe der Kompatibilität.

Dieser qualitative Aspekt der Kompatibilität erlaubt, die Paßgenauigkeit der Produktfunktionen mit den Bedürfnissen, Wünschen, Vorstellungen und Fähigkeiten des Nutzers zu beschreiben. Ausgehend von der niedrigsten Stufe, bei der ein Produkt nur funktioniert (die praktischen Funktionen erfüllt), über die Stufe, auf der es $g u t$, also effizient funktioniert bis zur höchsten Stufe, auf der es schön funk- tioniert. Der Nutzer freilich nennt es anders, beschreibt es als Geschmacksache, als Gefallen etc.

\subsection{Reaktions-Reaktions-Kompatibilität und Reiz- Reaktions-Kompatibilität}

Industriedesigner gestalten industriell fertigbare Produkte. Die Gestaltung der Produkte orientiert sich an den anatomischen, physiologischen und kognitiven Fähigkeiten der Nutzer. Dazu gehören auch Produkte, deren Gestaltung stark von physikalischen Gesetzen und ökonomischen Randbedingungen abhängt, wie beispielsweise Flugzeuge, die jedoch von menschlichen Passagieren genutzt bzw. von Menschen gehandhabt und gewartet werden müssen. Ergonomisches Wissen ist daher für Designer unabdingbar; Designer müssen die Eigenschaften und Fähigkeiten der Nutzer ihrer Produkte kennen. Dieses an Hochschulen in Ergonomiekursen vermittelte Wissen erlaubt, daß Produkte der Forderung nach Reaktions-Reaktions-Kompatibilität entsprechen, der Anpassung des Produktes an die motorischen Charakteristika des menschlichen Körpers. Am Beispiel eines Verkehrsflugzeuges ist das die Auslegung des Interieurs und der Notausgänge für die größten und die schwächsten Passagiere.

Die sinnesphysiologischen und kognitiven Eigenschaften werden in der Designausbildung - je nach Curriculum eher unter Themen wie Wahrnehmungspsychologie bzw. Interface-Design vermittelt.

Der Begriff Interface-Design beschreibt die Gestaltung von Bedienelementen und Anzeigen. Diese Elemente sind der Kommunikation verpflichtet. Sie müssen Auslöser und den zu erzielenden Effekt einander sinnfällig zuordnen, um im oben beschriebenen Sinn gut zu kommunizieren. Solche Interfaces sind idealerweise intuitiv verständlich bzw. weisen, in der von Strasser (1993) aufgestellten Systematik ausgedrückt, eine hohe Reiz-Reaktions-Kompatibilität auf. Sie verursachen geringen Umcodieraufwand, d. h. der Nutzer muß wenig interpretieren bzw. eine (erlernte) Bedeutung finden. Als Beispiel sei hier der Spurverlassenswarner im Fahrzeug angeführt. Verläßt das Fahrzeug die Spur, erinnert das System durch Vibration - die schneller umsetzbar ist, wenn sie nicht im Sitz, sondern im Lenkrad auftritt - an dem Bedienelement, mit dem eine Spurabweichung korrigiert wird.

Reiz-Reaktions-Kompatibilität wird erreicht, indem Richtungskompatibilität und räumliche Kompatibilität etc. gewährleistet werden. Viele dieser Forderungen entsprechen den im Design stark beachteten Gestaltgesetzen, die in der Gestaltpsychologie formuliert wurden. Sie beschreiben Gesetzmäßigkeiten der Wahrnehmung und der Bedeutungszuschreibung, die einer effizienten kognitiven Verarbeitung entsprechen. $\mathrm{Zu}$ diesen Prinzipien zählen die Figur-GrundOrganisation (Prägnanz), die gute Fortsetzung (Kontinuität) 


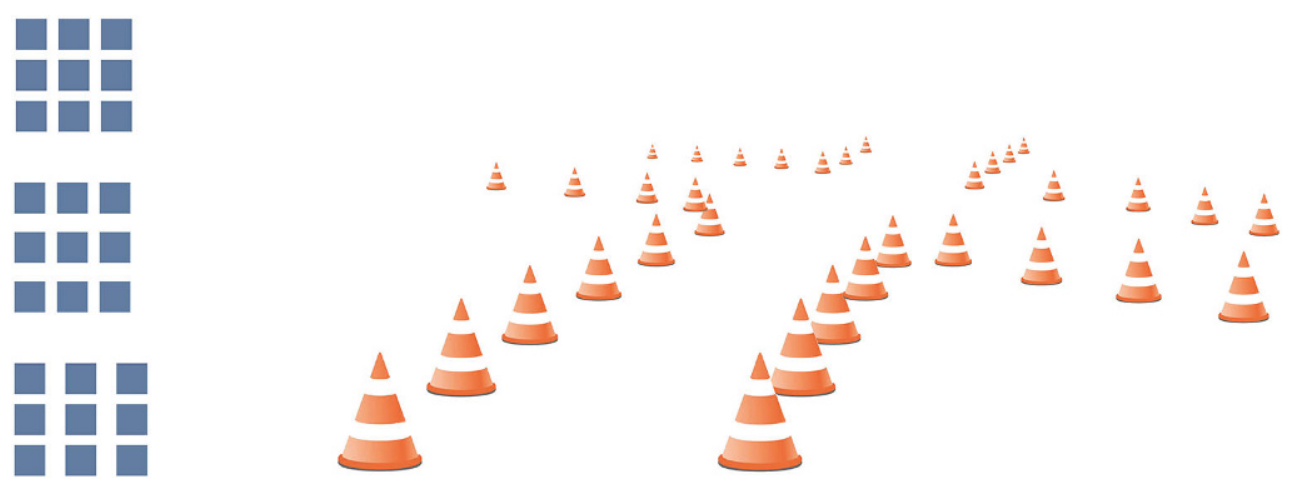

Abb. 5 Sog. Gestaltgesetze beschreiben, wie Einzelelemente im formalen Kontext sich gegenseitig beeinflussen. Nähe erzeugt Gruppen, hier Spalten bzw. Zeilen (links); eine virtuelle, aber erkennbare Reihung kann fortgesetzt werden (rechts)

Fig. 5 So called Gestalt principles describe the way single components affect each other in a formal context. Proximity turns single elements into groups, e.g. into columns and lines (left); a virtual albeit recognizable sequence can be continued (right)

und die Nähe (Gruppierung), etc. Die Nähe von Objekten beispielsweise beeinflußt, wie sie wahrgenommen werden, ob als einzelne Elemente oder als Gruppe (vgl. Abb. 5 links). Jeder, der sich schon fragte, ob er in einem Formular über oder unter der Angabe schreiben solle, traf auf wenig gruppierte und daher nicht zuzuordnende Elemente. Wie ist der mit Pylonen abgesteckte Parcours zu lesen? Geht es rechts oder links weiter? Sind die Pylonen zu weit voneinander entfernt, wird die sie verbindende Linie (gute Fortsetzung) nicht mehr erkannt (vgl. Abb. 5 rechts).

\subsection{Konzeptionelle Kompatibilität}

Im Interface-Design werden noch weitere Kompatibilitäten thematisiert. Die konzeptionelle Kompatibilität beschreibt beispielsweise, wie gut Codes und Symbole mit den Vorstellungen der Nutzer übereinstimmen und ob die verwendeten Codes für den Nutzer eine Bedeutung haben. Stimmt die Vorstellung des Nutzers, wie das Produkt funktioniert bzw. wie es zu bedienen ist, mit der tatsächlichen Funktionsweise und/oder Steuerung des Produktes überein, wirkt es selbstverständlich, intuitiv? Der kognitive Aufwand der Übersetzung entfällt damit.

Ein Steuerrad zum Beispiel scheint wie das Lenkrad eines Automobils zu funktionieren: Die Übersetzung auf die
Vorderräder bewirkt, daß die Lenkbewegung identisch mit der Richtungsänderung ist: Wohin ich lenke, dorthin fährt das Fahrzeug. Steigt der Autofahrer allerdings auf eine Jolle um, ist er überrascht, wenn er die Pinne legt und damit eine entgegengesetzte Richtungsänderung der Jolle bewirkt (vgl. Abb. 6).

Die Zuordnung wird durch eine Erwartung, eine modellhafte Vorstellung, das sog. mentale Modell, unterstützt. Stimmt das mentale Modell des Nutzers mit der Funktionsweise des unbekannten Produktes überein, kann er das Konzept des Bekannten analog auf das Unbekannte übertragen. Ignoriert der Designer allerdings das mentale Modell der Nutzer, führt das zu unnötiger Frustration bei der Verwendung des Produktes. Die Nutzer werden Schwierigkeiten haben, sich an die Bedienabläufe zu erinnern, da sie unlogisch erscheinen, nicht der eigenen Vorstellung von der Funktionsweise oder dem erwarteten Resultat entsprechen.

Die Nummer eines Hotelzimmers gibt Hinweise zur Lage des Zimmers. Je nach Höhe des Gebäudes steht die erste Ziffer bzw. stehen die ersten beiden Ziffern für das Stockwerk. Beim Aussteigen aus dem Lift trifft man auf einen Wegweiser, der auf den Gang deutet, in dem man das Zimmer findet - eine Entscheidungshilfe zur Laufrichtung (vgl. Abb. 7).

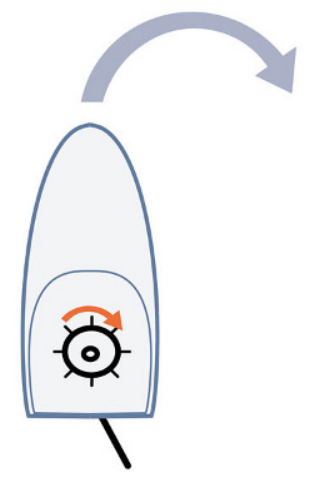

Abb. 6 Der Zusammenhang zwischen Lenkbewegung und Fahrzeugbewegung entspricht nicht immer den Erwartungen Fig. 6 The relationship between steering impulse and vehicle motion don't always meet the expectations

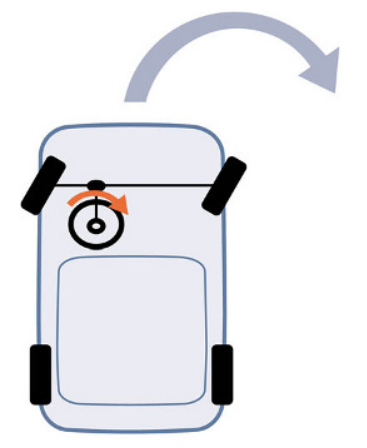




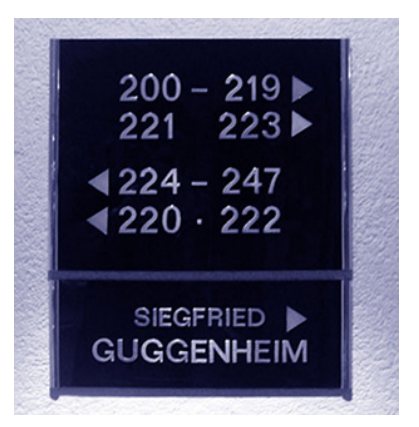

Abb. 7 Ein Wegweiser, der die Verteilung der Gästezimmer auf die Hotelgänge angibt

Fig. 7 Guide sign indicating the distribution of the guestrooms among the hotel corridors

Wir gehen davon aus, daß die Zimmernummern einem eindimensionalen Zahlenband entsprechen. Die Aufteilung der Zimmer auf die Gänge folgt jedoch häufig einer anderen Struktur. Hotelgäste, die ihr Zimmer als einen Punkt in einer aufsteigenden Zahlenfolge erwarten, sind dann gezwungen, die gesamte Anordnung zu verinnerlichen, oder eben doch die Gänge abzulaufen um ihr Zimmer zu finden.

\subsection{Kompatibilität der eingesetzten Modalitäten}

Kompatible Modalitäten erleichtern sowohl die Reaktion auf einen Reiz, als auch dessen kognitive Verarbeitung. Crossmodale Antworten gehen mit Wechselkosten einher, einer Einbuße in Antwortzeit oder -qualität. Die Kompatibilität von Modalitäten beschreibt einerseits den Einsatz des gleichen Sinneskanals für den Reiz wie für die Wahrnehmung der Reaktion, wie es beispielsweise die App Alexa eines sprachaktivierten Computers vormacht, die eine mündliche Antwort auf eine gehörte/mündlich gestellte Frage gibt. Andererseits wird eine kompatible Modalität auch durch die Art der Darstellung gekennzeichnet, wie z.B. bei einem Grafen mit beschrifteten Achsen im Gegensatz zu einer im Text beschriebenen Interpretation der Achsen (vgl. Abb. 8).

Faßt man die Schnittstelle zwischen Nutzer und einem Artefakt etwas weiter, ist jedes Produkt ein Interface, wie etwa die Sitzfläche des Stuhls oder der Griff des Quirls oder ein Smartphone. Die Oberfläche eines Produkts vermittelt zwischen dem Nutzer und dem Artefakt, vermittelt einerseits zwischen dem Wollen und Erwarten des Nutzers, andererseits zwischen der Funktionalität und dem Vermögen des Artefaktes.

Diese Vermittlung kann unter Einbeziehung aller Sinne stattfinden, z.B. als visuelle, akustische, taktile, olfaktorische und sensomotorische Kommunikation. Multimodale Interfaces ermöglichen eine Verteilung der Belastung einzelner Sinne. Meist konzentrieren wir uns auf einige wenige Sinneskanäle, ohne uns klar zu werden, daß meist mehrere Sinne bei der Kommunikation involviert sind. Soll dem menschlichen Gegenüber des Interfaces etwas angezeigt werden, wird oft reflexartig eine visuelle Anzeige eingesetzt. So bieten aktuelle Fahrzeug-Interieurs großflächige Touch-Screen-Vertäfelungen und fordern damit neben der Feinmotorik auch unseren Hauptsinn heraus, der eigentlich der primären Fahraufgabe gewidmet sein sollte.

Der Entwurf eines aktiven Gaspedals von Lukas Heintschel (2019) umgeht den beim Fahren ohnehin stark beanspruchten visuellen Sinn. Anstelle einer blinkenden Anzeige für die aktivierte Anti-Schlupf-Regelung kommuniziert dieses Gaspedal direkt mit dem Fuß des Fahrers. Es vermittelt zwischen der Beschaffenheit des Straßenbelags (Asphalt, Schotter, Eis, stehendes Wasser) und der Reaktion des Fahrers, indem es die Eigenschaften auf dem Gaspedal spiegelt und so die sonst durch Komfortmaßnahmen weggedämpften Impulse wiedergibt. Bei vereister Fahrbahn beispielsweise bewegt sich die Kontaktfläche auf dem Pedal leicht seitwärts und vermittelt den Eindruck, der Fuß rutsche auf der Pedalfläche. Intuitiv wird der Fuß sanfter aufgesetzt - eine Übersetzung in eine Handlung, die der kritischen Situation angemessen ist und den kognitiven Umweg vermeidet.
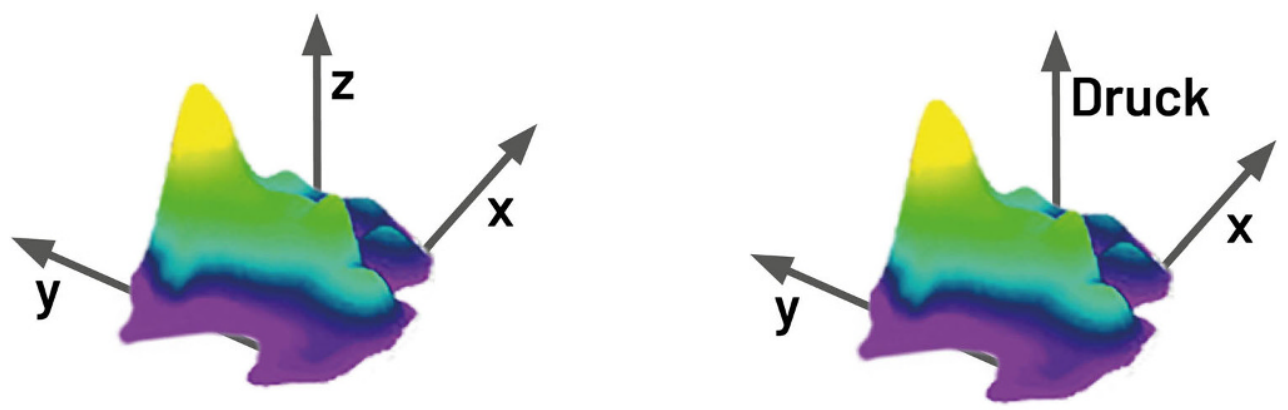

\section{z steht für Druck}

Abb. 8 Grafiken erläutern, was durch Worte oft weniger effektiv vermittelt werden kann. Der Vorteil direkter Anschaulichkeit wird verschenkt, wenn Elemente der Grafik mit Hilfe einer Beschreibung oder Legende übersetzt werden müssen

Fig. 8 Explanations offered by graphics often are more effective than by words. The benefits of a straightforward description are lost, when graphics elements have to be decoded via a separate description or a legend 


\section{Ein ganzheitlicher Blick auf die Begriffe Kompatibilität, Kontingenz, Konsistenz}

Die Entwicklung von Produkten, die zunehmend komplexer werden, erfordert eine ganzheitliche Sicht auf das Thema Kompatibilität. Immerhin kann Kompatibilität ein optimales Zusammenwirken von Produkten und ihren Nutzern ermöglichen. Da wir Produkte in unterschiedlichen Kontexten unterschiedlich wahrnehmen, prüfen Gestalter das Produkt aus unterschiedlichen Perspektiven und am besten auch aus unterschiedlichen Entfernungen, im Detail und aus großer Entfernung, als Teil eines Systems.

Wird beispielsweise ein Medizinprodukt entwickelt, prüft man die Perspektiven sämtlicher Stakeholder, die jeweils sehr unterschiedliche Anforderungen ergeben. Ärzte achten auf die praktische Funktionalität und die Verständlichkeit der Ergebnisse. Das Pflegepersonal wünscht sich intuitive Bedienung, Mobilität und Hygiene, das Servicepersonal gute Zugänglichkeit und das Reinigungspersonal Reinigungsfreundlichkeit. Der Krankenhausbetreiber erwartet Kompatibilität zu anderen Systemen. Der Entwickler versucht, innovative Technologien einzusetzen. Die Beschaffung rechnet mit schneller Amortisation. Die Logistiker fordern Kompatibilität mit Container- und Palettenmaßen. Der Produzent versucht, Gleichteile und eine vereinfachte Herstellung durchzusetzen. Der Vertrieb möchte beim Kunden mit neuen Funktionen werben. Besucher erwarten eine wohnliche Atmosphäre beim Krankenbesuch. Der Patient schließlich wünscht sich, gesund zu werden. Er möchte dabei weder von der Technologie eingeschüchtert noch zum Objekt der Behandler werden.

Wechselt man von dieser Betrachtung der Einzelinteressen zu einer ganzheitlichen Sicht, wird ein System sichtbar: Das Krankenhaus mit seinem Maschinenpark, das Umfeld, die Krankenhäuser mit eventuell ganz anderen Einsatzbedingungen. Wird ein fahrendes Krankenhaus im südamerikanischen Urwald dieses Gerät einsetzen? Welche Interessen haben die Stakeholder dort?

\subsection{Kulturelle Kompatibilität}

Kulturelle Kompatibilität kann sich auf viele Aspekte aufeinandertreffender Kulturen beziehen. Hierzu zählen nicht nur die sprachlichen Unterschiede zwischen Völkern, sozialen Gruppen und Zeiten, sondern auch unterschiedliche Werte und Ideale, Lebensstile, Umgangsformen, Sehgewohnheiten.

Am Beispiel eines Mißverständnisses zwischen einem Auftraggeber mit technischem Hintergrund und angehenden Designern wird deutlich, wie unterschiedliche Erfahrungen zu großen Differenzen in der Interpretation führen: Ein Gerät sollte an ein weiteres angehängt werden. Die

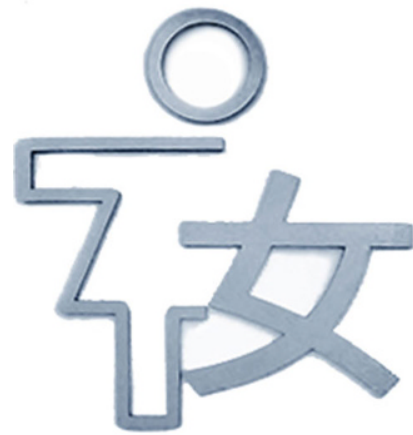

Abb. 9 Piktogramme vermeiden Sprachbarrieren. Da die Sehgewohnheiten sich dennoch kulturell unterscheiden, werden hier zwei Zeichen kombiniert, das im Westen geläufige Piktogramm für Frau und das aus einer bildlichen Darstellung einer Frau entwickelte chinesische Schriftzeichen (Ideogramm)

Fig. 9 Pictograms avoid language barriers. As habits of perception differ among cultures, this example combines the pictogram woman which is common in the West, and the respective Chinese character (ideogram), which was developed from a graphic representation of a woman

Elektrotechniker verstanden unter anhängen eine elektrische Verbindung, die Designer eine mechanische Kopplung.

Gerade bei international verwendeten Produkten greift man daher anstelle von Text eher zu bildlichen Darstellungen (vgl. Abb. 9).

Wir verstehen jedes Kulturprodukt als Ausdruck eines Inhalts, sei es das Licht einer Ampel, eine behandschuhte Hand mit Zigarettenspitze oder ein Buch - es wird als Kommunikation interpretiert. Mit dieser Zuordnung von Zeichen und Inhalten befaßt sich die Semantik. So sind die Erkenntnisse und Begriffe der Semantik zugleich die Grundlage der Diskussionen um Ausdruck und Eindruck in der Gestaltung: Wie bringe ich als Gestalter eine Botschaft mittels Artefakt an den Nutzer? Wie erwirke ich, daß das Produkt so aufgefaßt wird, wie es gemeint ist?

\subsection{Kontingenz}

Die Zuordnung von Zeichen und Bedeutung wird in der Gestaltung zwar intendiert, ist jedoch im praktischen Bezug mit Uneindeutigkeiten und Fehlinterpretationen behaftet. Das Gemeinte ist nicht das Ausgedrückte, das Wahrgenommene nicht zwingend das Verstandene. Die Mehrdeutigkeit besteht auf beiden Seiten, beim Sender und beim Empfänger der Botschaft: Die Botschaft des Senders ist kontingent und auch der vom Empfänger wahrgenommene Inhalt ist kontingent.

Niklas Luhmann (1987) definiert kontingent als ,etwas, was weder notwendig ist noch unmöglich ist; was also so, wie es ist (war, sein wird), sein kann, aber auch anders möglich ist." 

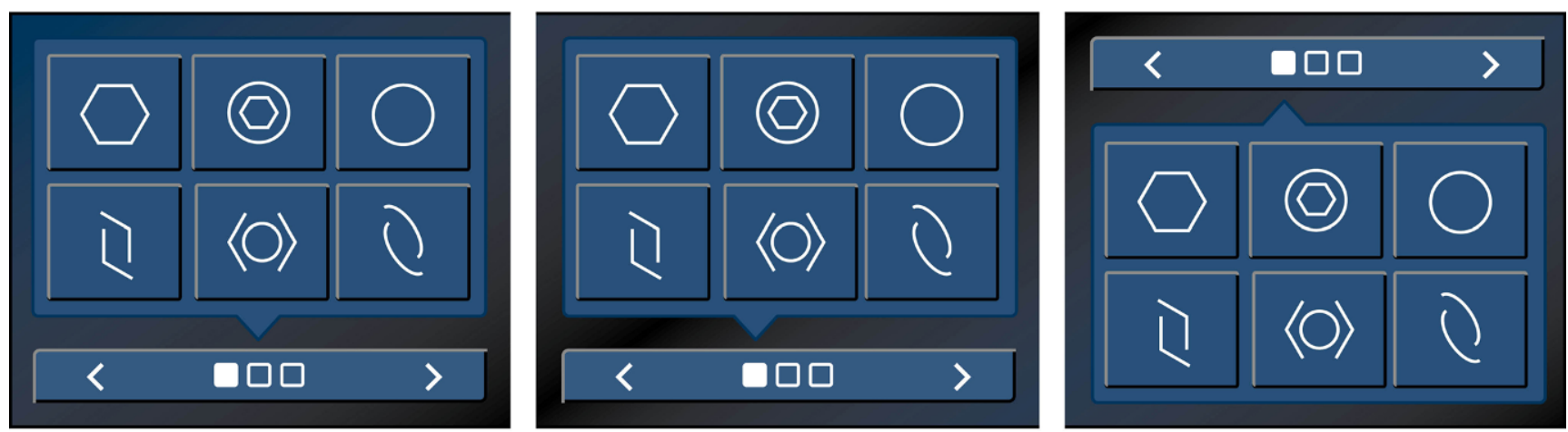

Abb. 10 Entwicklungsstufen eines Graphical User Interfaces (Lilian Dörr 2021): Die Grafik links faßt Elemente zusammen und verweist mit dem Sprechblasenende auf die weitere Information, die Seitenangabe. In der mittleren Grafik sind die beiden Anzeigen konsistenter, da das Sprechblasenende nicht nur auf die übergeordnete Struktur verweist, sondern zudem auf die aktuelle Position in dieser Struktur, die aktuelle Seite. In der Grafik rechts liegt die übergeordnete Struktur auch tatsächlich oben, entsprechen auch die sprachlichen und konzeptionellen Bilder der räumlichen Anordnung

Fig. 10 Development stages of a graphical user interface (Lilian Dörr 2021): The graphics on the left combine elements, the end of its speech bubble pointing at another information, the page indexing. The center graphics' two elements are more consistent, as the speech bubble is not only pointing at the superordinate structure, but to the actual position in that structure, the page number. In the graphics on the right the superordinate structure actually is placed on top, the linguistic and conceptional images correspond with the spatial layout

Kontingenz ist prinzipielle Offenheit und Ungewißheit menschlicher Lebenserfahrungen. Sie muß im Konzept der Kompatibilität schon mitgedacht werden, weil sie diese beeinflußt. Die Offenheit der Interpretation läßt keine eindeutige Kommunikation zu.

In der Konsequenz sind präzise, nicht interpretierbare Botschaften nicht möglich (Beispiel Gesetzestexte) und die Gewißheit, die intendierte Botschaft verstanden zu haben, ist irrational (Beispiel Liebesbrief).

Redundanz ist eine Art, die Kontingenz einer Botschaft zu verringern.

\subsection{Konsistenz}

Kompatibilität als Anschlußfähigkeit eines Systems zur erlebten Wirklichkeit wird nach Bubb (1993) in der Ergonomie als Äußere Kompatibilität bezeichnet. Ihr Gegenstück, die Innere Kompatibilität, bezeichnet die Sinnfälligkeit zwischen mentalem Modell und externer Information.

Für diese innere Stimmigkeit steht im Interface-Design der Begriff Konsistenz. Fehlt sie, kann der Nutzer die Erfahrung mit einem Element nicht auf ein anderes Element des gleichen Systems übertragen. Ein konsistentes System unterstützt den Nutzer, da er auf Gelerntes vertrauen kann. Er fühlt sich wohl damit, auch wenn neue Funktionen auftauchen.

Drückt das Produkt die Markenwerte aus? Entspricht die Gestaltung dem Habitus der Zeit? Drückt sie die Produktart und die Funktionsweise aus? Ist die Formensprache durchgängig? Welches mentale Modell wurde in anderen Elementen aufgebaut und wurde es hier angewandt? Entspricht diese Detaillösung der Gesamtanmutung? Konsistenz beginnt bei strategischen Überlegungen bis hin zu Detailfra- gen. Designer klopfen ihre Produkte auf Konsistenz ab und justieren sukzessiv Konzepte, Entwürfe und schließlich die Detaillösungen in kleinen Schritten (vgl. Abb. 10):

\section{Ausblick}

Kompatibilität wird zum Schlüsselbegriff der zukünftigen Produktentwicklung. Aufgrund zunehmender Komplexität kann man Produkte nicht mehr als stand-alone Objekte verstehen, sondern vielmehr als integrale Bestandteile eines Systems. Die Vernetzung der Produkte macht beispielsweise aus einem Fahrer samt seinem Fahrzeug einen vernetzten Verkehrsteilnehmer, der im Internet of Things per Smartphone, Bordcomputer, GPS und Internet mit anderen Verkehrsteilnehmern und Systemen kommuniziert. Die sich hieraus ergebende Komplexität bedarf einer höher- und weiterblickenden Perspektive, die über eine reine NutzerProdukt-Beziehung hinausgeht.

Kompatibilität stellt sicher, daß der Nutzer mit seinen Kompetenzen und Einschränkungen nicht nur eine gelingende Kommunikation erwarten kann, sondern auch eine gelingende Vernetzung mit anderen Systemen. Die Digitalisierung erspart dem Nutzer wohl viele Aufgaben, generiert aber auch neue Herausforderungen, die - sollte dieser Beitrag die Kompatibilität von Ergonomen und Designern fördern - in interdisziplinärer Zusammenarbeit zu bewältigen sind.

Die Perspektive des Designs bietet hierzu eine gute Ausgangsposition. Eine funktionale Optimierung der Produkte bedeutet nicht nur, eine effektive und effiziente Umsetzung der technisch-praktischen Anforderungen, sondern auch die Integration der Anzeichen- und Symbolfunktionen. 
Ästhetik, verstanden als die Verknüpfung von sinnlicher Wahrnehmung mit Bedeutungen, hat nicht nur etwas mit „schönen“ Produkten zu tun, sondern sie hilft, die Konsistenz und die Kompatibilität der Modalitäten sicherzustellen. Der Kulturphilosoph Bazon Brock sieht die Aufgabe der Ästhetik in der Vermittlung (Fohrbeck 1977). Die Disziplin Design greift diese Vermittlungsaufgabe auf und sorgt für eine erweiterte Kompatibilität zwischen Nutzer und Produkt.

Funding Open Access funding enabled and organized by Projekt DEAL.

Open Access Dieser Artikel wird unter der Creative Commons Namensnennung 4.0 International Lizenz veröffentlicht, welche die Nutzung, Vervielfältigung, Bearbeitung, Verbreitung und Wiedergabe in jeglichem Medium und Format erlaubt, sofern Sie den/die ursprünglichen Autor(en) und die Quelle ordnungsgemäß nennen, einen Link zur Creative Commons Lizenz beifügen und angeben, ob Änderungen vorgenommen wurden.

Die in diesem Artikel enthaltenen Bilder und sonstiges Drittmaterial unterliegen ebenfalls der genannten Creative Commons Lizenz, sofern sich aus der Abbildungslegende nichts anderes ergibt. Sofern das betreffende Material nicht unter der genannten Creative Commons Lizenz steht und die betreffende Handlung nicht nach gesetzlichen Vorschriften erlaubt ist, ist für die oben aufgeführten Weiterverwendungen des Materials die Einwilligung des jeweiligen Rechteinhabers einzuholen.

Weitere Details zur Lizenz entnehmen Sie bitte der Lizenzinformation auf http://creativecommons.org/licenses/by/4.0/deed.de.

\section{Literatur}

\section{Verwendete Literatur}

Bubb H (1993) Systemergonomische Gestaltung. Kap. 5.3. In: Schmidtke H (Hrsg) Ergonomie, 3. Aufl. Hanser, München, S 390-420
Dörr L (2021) Unveröffentlichter Interface-Entwurf für einen LKW, Hochschule München, Fakultät für Design

Fohrbeck K (Hrsg) (1977) Ästhetik als Vermittlung - Arbeitsbiographie eines Generalisten. DuMont, Köln

Hara K (2011) Designing design. Lars Müller Publishers, Zürich, S 411

Heintschel L (2019), unveröffentlichte Studienarbeit zum Thema Multisensualität, Hochschule München, Fakultät für Design

Jordan PW (2000) Designing pleasurable products. Taylor \& Francis, Boca Raton

Luhmann N (1987) Soziale Systeme - Grundriß einer allgemeinen Theorie. Suhrkamp, Frankfurt a. M., S 152

Steffen D (2000): Zur Theorie der Produktsprache, Kap 2. In: Design als Produktsprache: Der „Offenbacher Ansatz“ in Theorie und Praxis. Verlag form theorie, Frankfurt a. M., S 34-95

Strasser H (1993) Ergonomie - Arbeitsplatz Kompatibilität. Kap. 2.4.5. In: Hettinger T, Wobbe G (Hrsg) Kompendium der Arbeitswissenschaft. Kiel Verlag, Ludwigshafen, S 228-242

\section{Weiterführende Literatur}

Bonsiepe G (1996) Interface - Design neu begreifen. Bollmann, Mannheim

McDonagh D, Hekkert P, van Erp J, Gyi D (Hrsg) (2004) Design and Emotion-The Experience of Everyday Things. Taylor \& Francis, London

Mohs C (2011) Intuitive Benutzung technischer Systeme. Tectum, Marburg

Schmidtke H, Rühmann H (1981) Betriebsmittelgestaltung. Kap. 6.5. In: Schmidtke H (Hrsg) Lehrbuch der Ergonomie. Hanser, München, S 422-460

Schulz von Thun F (2011) Miteinander reden - Störungen und Klärungen. Allgemeine Psychologie der Kommunikation, 49. Aufl. Rowohlt, Reinbek 